\title{
Superconductivity and Superfluidity - Part III: Superfluidity as a Consequence of Ordering of Zero-point Oscillations
}

\author{
Boris V.Vasiliev \\ Independent Researcher, Russia \\ *Corresponding Author: bv.vasiliev@narod.ru
}

Copyright (C)2014 Horizon Research Publishing All rights reserved.

\begin{abstract}
Currently there is a common belief that the explanation of superconductivity phenomenon lies in understanding the mechanism of the formation of electron pairs. Paired electrons, however, cannot form a superconducting condensate spontaneously. These paired electrons perform disorderly zero-point oscillations and there are no force of attraction in their ensemble. In order to create a unified ensemble of particles, the pairs must order their zero-point fluctuations so that an attraction between the particles appears. As a result of this ordering of zero-point oscillations in the electron gas, superconductivity arises. This model of condensation of zero-point oscillations creates the possibility of being able to obtain estimates for the critical parameters of elementary superconductors, which are in satisfactory agreement with the measured data. On the another hand, the phenomenon of superfluidity in He-4 and He-3 can be similarly explained, due to the ordering of zero-point fluctuations. It is therefore established that both related phenomena are based on the same physical mechanism.
\end{abstract}

Keywords Superconductivity Superfluidity Zeropoint Oscillations

\section{Zero-point oscillations of $\mathrm{He}$ atoms and superfluidity}

The main features of superfluidity of liquid helium became clear few decades ago [1], [2]. L.D.Landau explains this phenomenon as the manifestation of a quantum behavior of the macroscopic object.

However, the causes and mechanism of the formation of superfluidity are not clear till our days. There is no explanation why the $\lambda$-transition in helium- 4 occurs at about $2 \mathrm{~K}$, that is about twice less than its boiling point:

$$
\frac{T_{\text {boiling }}}{T_{\lambda}} \approx 1.94
$$

while for helium-3, this transition is observed only at temperatures about a thousand times smaller.
The related phenomenon, superconductivity, can be regarded as superfluidity of a charged liquid. It can be quantitatively described considering it as the consequence of ordering of zero-point oscillations of electron gas. Therefore it seems appropriate to consider superfluidity from the same point of view.

Atoms in liquid helium-4 are electrically neutral, as they have no dipole moments and do not form molecules. Yet some electromagnetic mechanism should be responsible for phase transformations of liquid helium (as well as in other condensed substance where phase transformations are related to the changes of energy of the same scale).

F. London has demonstrated already in the 1930's [3], that there is an interaction between atoms in the ground state, and this interaction is of a quantum nature. It can be considered as a kind of the Van-der-Waals interaction. Atoms in their ground state $(\mathrm{T}=0)$ perform zero-point oscillations. F.London was considering vibrating atoms as three-dimensional oscillating dipoles which are connected to each other by the electromagnetic interaction. He proposed to call this interaction as the dispersion interaction of atoms in the ground state.

\section{The dispersion effect in inter- action of atoms in the ground state}

Following F.London [3], let us consider two spherically symmetric atoms without non-zero average dipole moments. Let us suppose that at some time the charges of these atoms are fluctuationally displaced from the equilibrium states:

$$
r_{1}=\left(x_{1}, y_{1}, z_{1}\right)
$$

$$
r_{2}=\left(x_{2}, y_{2}, z_{2}\right)
$$


If atoms are located along the Z-axis at the distance $L$ of each other, their potential energy can be written as:

$$
\begin{aligned}
\mathcal{H}= & \underbrace{\frac{e^{2} r_{1}^{2}}{2 a}}_{\text {elastic dipoles energy }}+\frac{\frac{e^{2} r_{2}^{2}}{2 a}}{e^{2}}+ \\
& +\underbrace{\frac{e^{2}}{L^{3}}\left(x_{1} x_{2}+y_{1} y_{2}-2 z_{1} z_{2}\right)}_{\text {elastic dipoles interaction }} .
\end{aligned}
$$

where $a$ is the atom polarizability.

The Hamiltonian can be diagonalized by using the normal coordinates of symmetric and antisymmetric displacements:

$$
r_{s} \equiv\left\{\begin{array}{l}
x_{s}=\frac{1}{\sqrt{2}}\left(x_{1}+x_{2}\right) \\
y_{s}=\frac{1}{\sqrt{2}}\left(y_{1}+y_{2}\right) \\
z_{s}=\frac{1}{\sqrt{2}}\left(z_{1}+z_{2}\right)
\end{array}\right.
$$

and

$$
r_{a} \equiv\left\{\begin{array}{l}
x_{a}=\frac{1}{\sqrt{2}}\left(x_{1}-x_{2}\right) \\
y_{a}=\frac{1}{\sqrt{2}}\left(y_{1}-y_{2}\right) \\
z_{a}=\frac{1}{\sqrt{2}}\left(z_{1}-z_{2}\right)
\end{array}\right.
$$

This yields

$$
\begin{aligned}
& x_{1}=\frac{1}{\sqrt{2}}\left(x_{s}+x_{a}\right) \\
& y_{1}=\frac{1}{\sqrt{2}}\left(y_{s}+y_{a}\right) \\
& z_{1}=\frac{1}{\sqrt{2}}\left(z_{s}+z_{a}\right)
\end{aligned}
$$

and

$$
\begin{aligned}
& x_{2}=\frac{1}{\sqrt{2}}\left(x_{s}-x_{a}\right) \\
& y_{2}=\frac{1}{\sqrt{2}}\left(y_{s}-y_{a}\right) \\
& z_{2}=\frac{1}{\sqrt{2}}\left(z_{s}-z_{a}\right)
\end{aligned}
$$

As the result of this change of variables we obtain:

$$
\begin{aligned}
& \mathcal{H}=\frac{e^{2}}{2 a}\left(r_{s}^{2}+r_{a}^{2}\right)+ \\
& +\frac{e^{2}}{2 L^{3}}\left(x_{s}^{2}+y_{s}^{3}-2 z_{s}^{2}-x_{a}^{2}-y_{a}^{2}+2 z_{a}^{2}\right)= \\
& =\frac{e^{2}}{2 a}\left[\left(1+\frac{a}{L^{3}}\right)\left(x_{s}^{2}+y_{s}^{2}\right)+\right. \\
& \quad+\left(1-\frac{a}{L^{3}}\right)\left(x_{a}^{2}+y_{a}^{2}\right)+ \\
& \left.\quad+\left(1-2 \frac{a}{L^{3}}\right) z_{s}^{2}+\left(1+2 \frac{a}{L^{3}}\right) z_{a}^{2}\right] .
\end{aligned}
$$

Consequently, frequencies of oscillators depend on their orientation and they are determined by the equations:

$$
\begin{aligned}
& \stackrel{s}{a}=\Omega_{0 y}^{a}=\Omega_{0} \sqrt{1 \pm \frac{a}{L^{3}}} \approx \\
& \approx \Omega_{0}\left(1 \pm \frac{a}{L^{3}}-\frac{a^{2}}{8 L^{6}} \pm \ldots\right) \\
& \stackrel{s}{a}=\Omega_{0} \sqrt{1 \mp \frac{2 a}{L^{3}}} \approx \\
& \approx \Omega_{0}\left(1 \mp \frac{a}{L^{3}}-\frac{a^{2}}{2 L^{6}} \mp \ldots\right),
\end{aligned}
$$

where

$$
\Omega_{0}=\frac{2 \pi e}{\sqrt{m a}}
$$

is natural frequency of the electronic shell of the atom (at $L \rightarrow \infty$ ). The energy of zero-point oscillations is

$$
\mathcal{E}=\frac{1}{2} \hbar\left(\Omega_{0}^{s}+\Omega_{0}^{a}\right)
$$

It is easy to see that the description of interactions between neutral atoms do not contain terms $\frac{1}{L^{3}}$, which are characteristics for the interaction of zero-point oscillations in the electron gas (Eq.(??)) and which are responsible for the occurrence of superconductivity.

The terms that are proportional to $\frac{1}{L^{6}}$ manifest themselves in interactions of neutral atoms.

It is important to emphasize that the energies of interaction are different for different orientations of zeropoint oscillations. So the interaction of zero-point oscillations oriented along the direction connecting the atoms leads to their attraction with energy:

$$
\mathcal{E}_{z}=-\frac{1}{2} \hbar \Omega_{0} \frac{A^{2}}{L^{6}}
$$

while the sum energy of the attraction of the oscillators of the perpendicular directions ( $\mathrm{x}$ and $\mathrm{y}$ ) is equal to one half of it:

$$
\mathcal{E}_{x+y}=-\frac{1}{4} \hbar \Omega_{0} \frac{A^{2}}{L^{6}}
$$

(the minus sign is taken here because for this case the opposite direction of dipoles is energetically favorable).

\section{The estimation of main char- acteristic parameters of super- fluid helium}

\subsection{The main characteristic parame- ters of the zero-point oscillations of atoms in superfluid helium-4}

There is no repulsion in a gas of neutral bosons. Therefore, due to attraction between the atoms at temperatures below

$$
T_{\text {boil }}=\frac{2}{3 k} \mathcal{E}_{z}
$$

this gas collapses and a liquid forms.

At twice lower temperature

$$
T_{\lambda}=\frac{2}{3 k} \mathcal{E}_{x+y}
$$

all zero-point oscillations become ordered. It creates an additional attraction and forms a single quantum ensemble.

A density of the boson condensate is limited by zero-point oscillations of its atoms. At condensation the distances between the atoms become approximately equal to amplitudes of zero-point oscillations.

Coming from it, we can calculate the basic properties of an ensemble of atoms with ordered zero-point oscillations, and compare them with measurement properties of superfluid helium.

We can assume that the radius of a helium atom is equal to the Bohr radius $a_{B}$, as it follows from quantummechanical calculations. Therefore, the energy of electrons on the s-shell of this atom can be considered to be equal:

$$
\hbar \Omega_{0}=\frac{4 e^{2}}{a_{B}}
$$


As the polarizability of atom is approximately equal to its volume [5]

$$
A \simeq a_{B}^{3},
$$

the potential energy of dispersive interaction (9), which causes the ordering zero-point oscillations in the ensemble of atoms, we can represent by the equation:

$$
\mathcal{E}_{x+y}=-\frac{e^{2}}{a_{B}} a_{B}^{6} n^{2}
$$

where the density of helium atoms

$$
n=\frac{1}{L^{3}}
$$

\subsubsection{The velocity of zero-point oscillations of helium atom}

It is naturally to suppose that zero-point oscillations of

atoms are harmonic and the equality of kinetic and potential energies are characteristic for them:

$$
\frac{M_{4}{\widehat{v_{0}}}^{2}}{2}-\frac{e^{2}}{a_{B}} a_{B}^{6} n^{2}=0,
$$

where $M_{4}$ is mass of helium atom, $\widehat{v_{0}}$ is their averaged velocity of harmonic zero-point oscillations.

Hence, after simple transformations we obtain:

$$
\widehat{v_{0}}=c \alpha^{3}\left\{\frac{n}{n_{0}}\right\}
$$

where the notation is introduced:

$$
n_{0}=\frac{\alpha^{2}}{a_{B}^{3}} \sqrt{\frac{M_{4}}{2 m_{e}}} .
$$

If the expression in the curly brackets

$$
\frac{n}{n_{0}}=1
$$

we obtain

$$
\widehat{v_{0}}=c \alpha^{3} \cong 116.5 \mathrm{~m} / \mathrm{s}
$$

\subsubsection{The density of liquid helium}

The condition (19) can be considered as the definition of the density of helium atoms in the superfluid state:

$$
n=n_{0}=\frac{\alpha^{2}}{a_{B}^{3}} \sqrt{\frac{M_{4}}{2 m_{e}}} \cong 2.172 \cdot 10^{22} \text { atom } / \mathrm{cm}^{3} .
$$

According to this definition, the density of liquid helium4

$$
\gamma_{4}=n M_{4} \cong 0.1443 \mathrm{~g} / \mathrm{cm}^{3}
$$

that is in good agreement with the measured density of the liquid helium $0.145 \mathrm{~g} / \mathrm{cm}^{3}$ for $T \simeq T_{\lambda}$.

Similar calculations for liquid helium-3 gives the density $0.094 \mathrm{~g} / \mathrm{cm}^{3}$, which can be regarded as consistent with its density $0.082 \mathrm{~g} / \mathrm{cm}^{3}$ experimentally measured near the boiling point.

\subsubsection{The dielectric constant of liquid helium}

To estimate the dielectric constant of helium we can use the Clausius-Mossotti equation [5]:

$$
\frac{\varepsilon-1}{\varepsilon+2}=\frac{4 \pi}{3} n A \text {. }
$$

At taking into account Eq.(13), we obtain

$$
\varepsilon \approx 1.040
$$

that differs slightly from the dielectric constant of the liquid helium, measured near the $\lambda$-point $[6]$ :

$$
\varepsilon \approx 1.057
$$

\subsubsection{The temperature of $\lambda$-point}

The superfluidity is destroyed at the temperature $T_{\lambda}$, at which the energy of thermal motion is compared with the energy of the Van-der-Waals bond in superfluid condensate

$$
\frac{3}{2} k T_{\lambda}-\frac{e^{2}}{a_{B}} a_{B}^{6} n^{2}=0
$$

With taking into account Eq.(21)

$$
T_{\lambda}=\frac{1}{3 k} \frac{M_{4}}{m_{e}} \frac{\alpha^{4} e^{2}}{a_{B}}
$$

or after appropriate substitutions

$$
T_{\lambda}=\frac{1}{3} \frac{M_{4} c^{2} \alpha^{6}}{k}=2.177 K
$$

that is in very good agreement with the measured value $T_{\lambda}=2.172 K^{1}$

\subsubsection{The boiling temperature of liquid helium}

After comparison of Eq.(8) - Eq.(9), we have

$$
T_{\text {boil }}=2 T_{\lambda}=4.35 \mathrm{~K}
$$

This is the basis for the assumption that the liquefaction of helium is due to the attractive forces between the atoms with ordered lengthwise components of their oscillations.

\subsubsection{The velocity of the first sound in liquid helium}

It is known from the theory of the harmonic oscillator that the maximum value of its velocity is twice bigger than its average velocity. In this connection, at assumption that the first sound speed $c_{s 1}$ is limited by this maximum speed oscillator, we obtain

$$
c_{s 1}=2 \widehat{v_{0}} \simeq 233 \mathrm{~m} / \mathrm{s}
$$

It is in consistent with the measured value of the velocity of the first sound in helium, which has the

\footnotetext{
${ }^{1}$ There is a unexpected fact. The expression (28) for the temperature of $\lambda$-transition is given without any explanations in some articles of Internet at citing of patents [7]. These articles and patents say nothing at all about zero-point oscillations, and don't give generally any explanations of the reasons that allowed to write this expression.
} 
maximum value of $238.3 \mathrm{~m} / \mathrm{s}$ at $T \rightarrow 0$ and decreases with increasing temperature up to about $220 \mathrm{~m} / \mathrm{s}$ at $T=T_{\lambda}$.

The results obtained in this section are summarized for clarity in the Table.(1).

The measurement data in this table are mainly quoted by [4] and [6].

\subsection{The estimation of characteristic properties of $\mathrm{He}-3$}

The results of similar calculations for the helium-3 properties are summarized in the Tab.(2).

There is a radical difference between mechanisms of transition to the superfluid state for He-3 and He- 4 . Superfluidity occurs if complete ordering exists in the atomic system. For superfluidity of He-3 electromagnetic interaction should order not only zero-point vibrations of atoms, but also the magnetic moments of the nuclei.

It is important to note that all characteristic dimensions of this task: the amplitude of the zero-point oscillations, the atomic radius, the distance between atoms in liquid helium - all equal to the Bohr radius $a_{B}$ by the order of magnitude. Due to this fact, we can estimate the oscillating magnetic field, which a fluctuating electronic shell creates on "its" nucleus:

$$
H_{\Omega} \approx \frac{e}{a_{B}^{2}} \frac{a_{B} \Omega_{0}}{c} \approx \frac{\mu_{B}}{A_{3}}
$$

where $\mu_{B}=\frac{e \hbar}{2 m_{e} c}$ is the Bohr magneton, $A_{3}$ is the electric polarizability of helium-3 atom.

Because the value of magnetic moments for the nuclei He-3 is approximately equal to the nuclear Bohr magneton $\mu_{n_{B}}=\frac{e \hbar}{2 m_{p} c}$, the ordering in their system must occur below the critical temperature

$$
T_{c}=\frac{\mu_{n_{B}} H_{\Omega}}{k} \approx 10^{-3} K
$$

This finding is in agreement with the measurement data. The fact that the nuclear moments can be arranged in parallel or antiparallel to each other is consistent with the presence of the respective phases of superfluid helium-3.

Concluding this approach permits to explain the mechanism of superfluidity in liquid helium.

In this way, the quantitative estimations of main parameters of the liquid helium and its transition to the superfluid state were obtained.

It was established that the phenomenon of superfluidity as well as the phenomenon of superconductivity is based on the physical mechanism of the ordering of zero-point oscillations.

\section{Conclusions}

Until now it has been commonly thought that the existence of the isotope effect in superconductors leaves only one way for explanation of the superconductivity phenomenon - the way based on the phonon mechanism.

Over fifty years of theory development based on the phonon mechanism, has not lead to success. All attempts to explain why some superconductors have certain critical temperatures (and critical magnetic fields) have failed.

This problem was further exacerbated with the discovery of high temperature superconductors. How can we move forward in HTSC understanding, if we cannot understand the mechanism that determines the critical temperature elementary superconductors?

In recent decades, experimenters have shown that isotopic substitution in metals leads to a change in the parameters of their crystal lattice and thereby affect the Fermi energy of the metal. As results, the superconductivity can be based on a nonphonon mechanism.

The theory proposed in this paper suggests that the specificity of the association mechanism of electrons pairing is not essential. It is merely important that such a mechanism was operational over the whole considered range of temperatures. The nature of the mechanism forming the electron pairs does not matter, because although the work of this mechanism is necessary it is still not a sufficient condition for the superconducting condensate's existence. This is caused by the fact that after the electron pairing, they still remain as non-identical particles and cannot form the condensate, because the individual pairs differ from each other as they commit uncorrelated zero-point oscillations. Only after an ordering of these zero-point oscillations, an energetically favorable lowering of the energy can be reached and a condensate at the level of minimum energy can then be formed. Due to this reason the ordering of zero-point oscillations must be considered as the cause of the occurrence of superconductivity.

Therefore, the density of superconducting carriers and the critical temperature of a superconductor are determined by the Fermi energy of the metal, The critical magnetic field of a superconductor is given by the mechanism of destruction of the coherence of zero-point oscillations.

In conclusion, the consideration of zero-point oscillations allows us to construct the theory of superconductivity, which is characterized by the ability to give estimations for the critical parameters of elementary superconductors. These results are in satisfactory agreement with measured data.

This approach permit to explain the mechanism of superfluidity in liquid helium. For electron shells of atoms in s-states, the energy of interaction of zero-point oscillations can be considered as a manifestation of Vander-Waals forces. In this way the opposite quantitative estimations of temperatures of the helium liquefaction and its transition to the superfluid state was obtained.

Thus it is established that both related phenomena, superconductivity and superfluidity, are based on the same physical mechanism - they both are consequences of the ordering of zero-point oscillations. 
Table 1. Comparison of the calculated values of liquid helium-4 with the measurement data

\begin{tabular}{|c|c|c|c|}
\hline parameter & $\begin{array}{l}\text { defining } \\
\text { formula }\end{array}$ & $\begin{array}{l}\text { calculated } \\
\text { value }\end{array}$ & $\begin{array}{c}\text { measured } \\
\text { value }\end{array}$ \\
\hline $\begin{array}{c}\text { the velocity of zero-point } \\
\text { oscillations of } \\
\text { helium atom }\end{array}$ & $\widehat{v_{0}}=c \alpha^{3}$ & $\begin{array}{c}116.5 \\
\mathrm{~m} / \mathrm{s}\end{array}$ & \\
\hline $\begin{array}{c}\text { The density of atoms } \\
\text { in liquid } \\
\text { helium }\end{array}$ & $n=\sqrt{\frac{M_{4}}{2 m_{e}}} \frac{\alpha^{2}}{a_{B}^{3}}$ & $\begin{array}{l}2.172 \cdot 10^{22} \\
\text { atom } / \mathrm{cm}^{3}\end{array}$ & \\
\hline $\begin{array}{c}\text { The density } \\
\text { of liquid helium-4 } \\
g / l\end{array}$ & $\gamma=M_{4} n$ & 144.3 & $145_{T \simeq T_{\lambda}}$ \\
\hline $\begin{array}{c}\text { The dielectric } \\
\text { constant } \\
\text { of liquid helium-4 }\end{array}$ & $\frac{\varepsilon-1}{\varepsilon+2}=\frac{4 \pi}{3} \alpha^{2} \sqrt{\frac{M_{4}}{2 m_{e}}}$ & 1.040 & $\begin{array}{l}1.048_{T \simeq 4.2} \\
1.057_{T \simeq T_{\lambda}}\end{array}$ \\
\hline $\begin{array}{c}\text { The temperature } \\
\lambda \text {-point, } \mathrm{K}\end{array}$ & $T_{\lambda} \simeq \frac{M_{4} c^{2} \alpha^{6}}{3}$ & 2.177 & 2.172 \\
\hline $\begin{array}{c}\text { The boiling } \\
\text { temperature } \\
\text { of helium-4,K } \\
\end{array}$ & $T_{b o i l} \simeq 2 T_{\lambda}$ & 4.35 & 4.21 \\
\hline $\begin{array}{l}\text { The first sound } \\
\text { velocity, } \\
\mathrm{m} / \mathrm{s}\end{array}$ & $c_{s 1}=2 \widehat{v_{0}}$ & 233 & $238.3_{T \rightarrow 0}$ \\
\hline
\end{tabular}

Table 2. The characteristic properties of liquid helium-3

\begin{tabular}{||c|c|c||c||}
\hline \hline parameter & defining & calculated & measured \\
formula & value & value \\
\hline $\begin{array}{c}\text { The velocity of zero-point } \\
\text { oscillations of } \\
\text { helium atom }\end{array}$ & $\widehat{v}_{0}=c \alpha^{3}$ & $\begin{array}{c}116.5 \\
\mathrm{~m} / \mathrm{s}\end{array}$ & \\
\hline \hline $\begin{array}{c}\text { The density of atoms } \\
\text { in liquid } \\
\text { helium-3 }\end{array}$ & $n_{3}=\sqrt{\frac{M_{3}}{2 m_{e}} \frac{\alpha^{2}}{a_{B}^{3}}}$ & $\begin{array}{c}1.88 \cdot 10^{22} \\
\text { atom/cm }\end{array}$ & \\
\hline \hline $\begin{array}{c}\text { The density } \\
\text { of liquid } \\
\text { helium-3, } \mathrm{g} / \mathrm{l}\end{array}$ & $\gamma=M_{3} n_{3}$ & 93.7 & 82.3 \\
\hline $\begin{array}{c}\text { The dielectric } \\
\text { constant } \\
\text { of liquid helium-3 }\end{array}$ & $\frac{\varepsilon-1}{\varepsilon+2}=\frac{4 \pi}{3} \alpha^{2} \sqrt{\frac{M_{3}}{2 m_{e}}}$ & 1.035 & \\
\hline \hline $\begin{array}{c}\text { The boiling } \\
\text { temperature } \\
\text { of helium-3,K }\end{array}$ & $T_{b o i l} \simeq \frac{4}{3} \frac{\mathcal{E}_{x+y}}{k}$ & 3.27 & 3.19 \\
\hline $\begin{array}{c}\text { The sound velocity } \\
\text { in liquid } \\
\text { helium-3 }\end{array}$ & $c_{s}=2 \widehat{v_{0}}$ & 233 & \\
\hline \hline
\end{tabular}




\section{REFERENCES}

[1] Landau L.D. : JETP, 11, 592 (1941)

[2] Khalatnikov I.M.: Introduction into theory of superfluidity, Moscow, Nauka, (1965)

[4] Kikoine I.K. a.o.: Physical Tables, Moscow, Atomizdat (1978) (in Russian).

[5] Fröhlich H. : Theory of dielectrics, Oxford, (1957)

[6] Russel J.Donnelly and Carlo F.Barenghy: The Observed Properties of Liquid Helium, Journal of Physical and Chemical Data, 6, N1, pp.51-104, (1977)

[7] Ilianok A.M: Eurasian patent 003164, US patent 6,570,224B1, Korean patent N 10-0646267, China patent CN 1338120.

[3] London F.: Trans. Faraday Soc. 33, p.8 (1937) 